\title{
A numerical method for simulating fluid flow through 3-D fracture networks
}

\author{
Na Huang ${ }^{a, b}$, Yujing Jiang ${ }^{b, a^{*}}$, Bo $\mathrm{Li}^{c}$, Richeng Liu ${ }^{d, a}$
}

${ }^{a}$ School of Engineering, Nagasaki University, 1-14 Bunkyo-machi, 8528521 Nagasaki, Japan

${ }^{b}$ State Key Laboratory of Mining Disaster Prevention and Control Co-founded by Shandong Province and the Ministry of Science and Technology, Shandong University of Science and Technology, Qingdao 266590, P.R.

China

${ }^{c}$ Rock Mechanics and Geo-Hazards Center, Shaoxing University, Shaoxing 312000, P.R. China

${ }^{d}$ State Key Laboratory for Geomechanics and Deep Underground Engineering, China University of Mining and Technology, Xuzhou 221116, P.R. China

* Corresponding author: Yujing Jiang Tel.: +81 95-819-2612

E-mail address: jiang@nagasaki-u.ac.jp (Y. Jiang) 
Abstract: Hydraulic property of fractured rock masses is commonly undertaken based on 2-D fracture network models, which are cut planes of the real 3-D models. This simplification would lead to a significant underestimation of fracture network permeability. In this study, a numerical procedure is originally developed to address flow problem through 3-D discrete fracture network (DFN) models. In this method, fractures are modeled as circular discs with arbitrary size, orientation and location. Fracture networks are established with fractures following well-known statistical distributions, after which the networks are triangulated and fluid flow is calculated by solving the Reynolds equation using Galerkin method. The results show that the permeability of 2-D DFN models that are cut from an original 3-D DFN model would be underestimated by 19.2 43.6\%, comparing with that of the 3-D DFN model. For networks that are consisted of power-law size-distributed fractures, the equivalent permeability would decrease exponentially with the increasing length distribution exponent. This tendency can be interpreted by incorporating the average intersection length, which is a parameter that can reflect the connectivity of a fracture network. When the heterogeneity of fracture aperture distribution is considered, some tortuous flow paths are formatted in 3-D fracture networks. The rougher fracture surface, the stronger anisotropy of aperture distribution, and thereby resulting in the larger reduction of the network permeability.

Key words: Discrete fracture network, flow simulation, Galerkin method, permeability 


\section{Introduction}

Accurate description and quantitative estimation of permeability of fractured rock masses have attracted substantial attentions in the past few decades in various perspectives such as radioactive waste storage, geothermal energy extractions, oil and gas production including lately the nonconventional shale gas resources in hard-rock aquifers [Lee et al. 2003; Neuman et al. 2005; De Dreuzy et al. 2013; Cai et al. 2013a]. Permeability of a rock mass is mainly governed by fractures and rock matrix [Lang et al. 2014; Wei et al. 2015; Cai et al. 2015; Chen et al. 2015b]. However, since fractures are more permeable than the matrix, they are recognized as the predominant pathways of fluid flow and solute transport [De Dreuzy et al. 2012; Ishibashi et al. 2012; Liu et al. 2016a]. In engineering practices, owing to the computational limitations and the insufficient 3-D geological information, analysis of hydraulic properties of rock fractures is often reduced to a 2-D problem [Nick et al. 2011; Rutqvist et al. 2013; Lang et al. 2014]. In recent years, a great amount of efforts has been exerted to study flow characteristic of 2-D fracture networks, and significant progress has been made towards the theoretical modeling, laboratory experiments and numerical methods [Hull et al. 1987; Min et al. 2004; Baghbanan et al. 2007; Zhao et al. 2011; Liu et al. 2016b]. However, natural fractured media usually displays a strong hydraulic complexity coming from the internal topography of fractures (i.e., geometry of the void spaces within single fracture) and from their arrangement in complex networks (i.e., geometry of the fracture network) [Bear et al. 2012; De Dreuzy et al. 2012, 2013; Cai et al. 2014]. The 2-D fracture model, which is a cut plane of the 3-D fracture network, cannot capture the geometry of real features. Whereas 3-D fracture networks have the outstanding advances of describing the orientation, connectivity, and permeability tensor of the real rock masses [Gonzalez-Garcia et al. 2000; Bogdanov et al. 2003; Ivanova et al. 2014; Hyman et al. 2015]. Recently, Lang et al. [2014] compared the equivalent permeability of 3-D fractured rock mass with the permeability computed on arbitrarily extracted 2-D cut planes. They found that the latter would underestimate the magnitude of permeability by up 
to 3 orders of magnitude. Therefore it is necessary to develop efficient and systematic methods for identifying the hydraulic properties of 3-D fracture networks.

The history of 3-D discrete fracture models goes more than twenty years, with fracture networks approximated to be restricted to idealized channels within the fracture plane [Long et al. 1985; Cacas et al. 1990]. This simplification allows for fast calculations with large-scale fracture networks, however the model is too simple to capture the complex flow behavior. Andersson and Dverstorp [1987] used a boundary element method to solve flow through each fracture. The flow characteristics in fractures can be detailedly described, whereas the number of fracture in the model is very limited. Koudina et al. [1998] discretized the stationary Reynolds equation on the triangular mesh by means of a finite-volume formulation to solve the flow problem, however the numerical applications are restricted to networks that are supposed to be statistically homogenous. Later many other numerical modeling methods have been continuously proposed [Mustapha et al. 2007; Ishibashi et al. 2012], however those methods usually have generic limitations: the intersections of fractures are usually either not considered or highly simplified. This may underestimate the role of intersections in the total hydraulic behavior, since intersections are strongly correlated to the fracture connectivity that is the first-order determinant of the occurrence of flow[De Dreuzy et al. 2000]. Nœtinger (2015) used a quasi-steady state method to solve transient Darcy flow in 3D fractured networks that assumed the characteristic diffusion time over one single fracture is negligible compared with the characteristic time of the macroscopic problem. Zhang (2015) proposed an approach for triangulating arbitrary loops and calculated the equivalent permeability for some complex DFNs. Benedetto et al. (2016) introduced a new approach for flow simulations in which the Virtual Element Method was used in handling polygonal meshes and only weak continuity is required for the hydraulic head along the intersections between fractures. However, in those methods, individual fractures were all characterized by a single aperture value, and the effects of the heterogeneity of aperture distributions on 3-D fluid flow cannot be considered.

In analyzing fluid flow through fracture networks, discrete fracture network (DFN) approach is 
a widely used modeling technique [De Dreuzy et al. 2012; Zhang et al. 2014; Hyman et al. 2015]. In contrast to continuum methodologies which are mainly applied for flow analyses over a lager domain, DFN approach is better treated in explicit representation of fracture geometries (fracture length, density, location, orientation and aperture) together with specific description of their intersections [Ishibashi et al. 2012]. Whereas fracture systems are usually geometrically complex, and the usual practice in DFN modeling assumed that the geometry of fractures was statistically distributed [Andersson et al. 1987; Jing et al. 2000]. Thus a prerequisite of modeling consists in determining how fracture geometries influence their hydraulic properties.

In the present study, a numerical procedure is originally developed to address flow problem through 3-D DFN models with fractures treated as circular discs with arbitrary size, orientation and location. The developed method was used to conduct a comparison of permeability between a 3-D DFN model and a series of 2-D cut planes. Then the effects of fracture length distribution and fracture heterogeneity on the equivalent permeability of 3-D DFN model were investigated. Relations between equivalent permeability and the geometrical properties of 3-D DFN were analyzed.

\section{Geological characteristics of 3-D DFN}

Natural fractured media has a broad range of scales from millimeters to kilometers [Cai et al. 2013b; Lei et al. 2015]. Since the minor structures of fracture usually cannot be completely mapped from borehole or exposure, it is usually difficult to obtain the complete geometrical data for a deterministic model. Therefore stochastic approaches have been widely used to address the uncertainty in DFNs by generating fracture networks where the characteristics such as fracture length and orientation follow some given probability distributions [Erhel et al. 2009; Hyman et al. 2015]. Based on a large number of field investigations and trace data analysis, Baecher et al. [1978] and Long et al. [1985] found that fractures can be primarily characterized by shapes of circle and/or ellipse. In the models presented in this study, all the fractures are assumed to be circular disks, with 
the center location uniformly distributed. The fracture density, length-distribution, and orientation are three of the most critical features that control the hydraulic properties of DFNs. As for the density of fractures, different methods according to available data in the field have been used in previous studies. Among them $P_{32}$ is a classical parameter that is defined as the total area fracture surface per unit volume [Davy et al. 2006b].

Several studies [De Dreuzy et al. 2000; Berkowitz 2002; Darcel et al. 2003b] have reported that the fracture length, which corresponds to the diameter of disk in this study, is usually broadly-ranged and exhibits no characteristic length scales. The length distribution of fractures can be correctly modeled using a power-law function as

$$
n(l)=\alpha \cdot l^{-a}
$$

where $n(l) \cdot d l$ is the number of fractures having a length in the range $[l, l+d l], \alpha$ is the coefficient of proportionality that represents the total amount or density of fractures by the range of fracture length, and $a$ is the power law exponent varying generally between 2.0 and 4.5 [De Dreuzy et al. 2012]. The lager value of $a$ would lead to the generation of a group of smaller fractures. The distribution of fracture length $l$ is defined between $l_{\min }$ and $l_{\max }$, which are the lower and upper bounds of the fracture lengths, respectively.

Another important parameter of DFN is fracture orientation. The fracture orientation is generally defined by dip $\theta_{1}$ and dip direction $\theta_{2}$. Since the fracture orientation may form clusters or sets around one or more statistically preferred directions in a rock mass, each set should be defined individually. The fisher distribution is used to model the preferential orientations with a given dispersion around the mean value as [Fisher et al. 1996]

$$
f(\theta)=\frac{\kappa \sin \theta e^{\kappa \cos \theta}}{e^{\kappa}-e^{-\kappa}}
$$

where $\theta$ is the angular deviation from the mean vector, and $\kappa$ is the Fisher constant or the dispersion factor.

A 3-D DEM (discrete element method) code 3DEC was adopted as the basic numerical tool for the geometric modeling of DFN. The fractures are generated with characteristics following the 
predetermined probability distributions. Then isolated fractures would be deleted as they do no contribution to fluid flow. For simulation purposes, a cubic computational domain $\Omega$ of characteristic size $L$ is introduced as performed in Erhel's study [2009]. The domain is composed of all fractures $\Omega_{f}$ with $f=1, \ldots N F$, and fracture intersection segment $S_{i}$ with $i=1, \ldots N I$, where $N F$ is the total number of fractures and $N I$ is the total number of intersection segments. The boundary of $\Omega$ is composed of the borders of the domain and the edges of the fractures. The boundary of fracture is composed of the circle edge, intersection lines with other fractures and intersection lines connected with the domain faces.

\section{Mesh generation}

Since fractures have circular shapes and could be intersected with each other at various angles and positions, a DFN model may yield very complicated fracture connections, especially when fractures are dense. Therefore, triangulation is assumed to be the most natural discretization. Whereas several constrains should be applied to this triangulation. At network scale, fractures that intersect directly or indirectly with inflow/outflow boundaries could form the flow path and fluid flows from one fracture to another through their intersection lines. Therefore the intersection between fractures in flow paths control the whole flow scheme. And the consistency of the meshes in intersecting lines is a prerequisite in order to ensure the correct connections of the flow element. So in the 3-D space, the intersection lines should be uniquely discretized with 3-D coordinates, which means that the nodes on such line should match with the nodes on each intersecting fracture (as shown in Fig. 1). At the fracture scale, each plane should be 2-D discretized, in order to guarantee mass conservation in each fracture plane. These are common requirements in the field [Koudina et al. 1998; Erhel et al. 2009; De Dreuzy et al. 2012]. One more constrain is applied in this study: the intersection lines are all extended to the fracture border, with the fracture plane divided into several irregular regions. In Fig. 1, the red lines are actual intersection lines, and the 
green lines are their corresponding extensions. This is aimed to handle continuity conditions of head and flux on intersections, which would be interpreted in the next section.

Mesh generation is still a challenging work especially with the requirement of a unique definition of fracture intersections which are arbitrarily distributed in each fracture face [Erhel et al. 2009; Lang et al. 2012; Zhang et al. 2015]. In this study the discretization of fracture network to triangular meshes would be performed with the help of a mesh generation code in 3DEC. In 3DEC fractures are necessarily extended to cut the computational domain into a combination of small blocks. Then tetrahedral-triangular element meshes on computational domain are performed with fractures represented as surfaces. A FISH program is developed to abstract the elements on fracture planes. Since the nodes are not exactly generated on the fracture circular border, the circular fracture area would be simplified to be a polygon area with the nodes nearest the fracture border combined together. This simplification is generally reasonable since the fracture shape in nature is not exactly circular and many studies characterized the fracture shape as polygon [Koudina et al. 1998; Khamforoush et al. 2008].

\section{Fluid flow through 3-D DFNs}

\subsection{Governing equations}

The fluid flow through fractures is governed by the nonlinear Navier-Stokes equations. However, due to the enormous difficulties of solving the Navier-Stokes equations composed of a set of coupled nonlinear partial derivatives of varying orders, most previous works assumed that the cubic law is applicable [Liu et al. 2015], where the cubic law is expressed as $Q=-b_{h}{ }^{3} \nabla P /(12 \mu)$, where $Q$ is the flow rate, $b_{h}$ is the hydraulic aperture, $P$ is the pressure, and $\mu$ is viscosity of the fluid. In order to describe the flow equation in each fracture, a projection from the 3-D coordinate system onto the fracture plane is defined. The coordinates of a node in 3-D system were represented by $(x$, $y, z)$, and the projected coordinates in a fracture plane $\Omega_{f}$ were represented by $\left(x_{f}, y_{f}\right)$. The governing 
equation in each fracture $\Omega_{f}$ can be written as:

$$
\frac{\partial}{\partial x_{f}}\left(K \frac{\partial h}{\partial x_{f}}\right)+\frac{\partial}{\partial y_{f}}\left(K \frac{\partial h}{\partial y_{f}}\right)=0
$$

where $h$ is the hydraulic head and $K$ is the fracture permeability which is closely related to fracture apertures in $\Omega_{f}$. This equation, known as Reynolds equation, can be used to study the flow through rough fractures [Brown 1987; Koyama et al. 2009]. The approach is to divide the void space between the two fracture surfaces into a great number of small simplified parallel-plates. Then the geological roughness of the fracture can be incorporated into the model by assignation of spatially variable apertures to fracture elements. Therefore, the local permeability can be determined element by element thus obtaining a random flow field.

The classical permeameter boundary conditions on the cube faces are applied: two opposite faces $\left(\Gamma_{\text {in }}\right.$ and $\left.\Gamma_{\text {out }}\right)$ of the model have fixed heads $h_{1}$ and $h_{2}$, and other four faces $\left(\Gamma_{\text {imp }}\right)$ are impermeable, and a Neumann zero flux condition on each fracture edge $\Gamma_{f}$, which can be summarized as

$$
\left\{\begin{array}{c}
h=h_{1} \quad \text { on } \Gamma_{f} \cap \Gamma_{\text {in }} \\
h=h_{2} \quad \text { on } \Gamma_{f} \cap \Gamma_{\text {out }} \\
(\nabla h) \cdot \mathbf{n}=0 \text { on } \Gamma_{f} \cap \Gamma_{\text {imp }} \\
(\nabla h) \cdot \mathbf{n}=0 \text { on } \Gamma_{f}
\end{array}\right.
$$

Continuity conditions in each fracture intersection lines are [Erhel et al. 2009]

$$
\left\{\begin{array}{l}
h_{k, f}=h_{k} \text { on } E_{k}, \forall f \in F_{k} \\
\sum_{f \in F_{k}} v_{k, f} \cdot n_{k, f}=0 \text { on } S_{k}
\end{array}\right.
$$

where $h_{k, f}$ is the trace of the head, $v_{k, f}$ is the flow velocity and $n_{k, f}$ is the normal unit vector on the boundary $S_{k}$ of the fracture $\Omega_{f}$.

\subsection{Numerical solution procedure}

A program based on the Galerkin method is originally developed to solve the flow through 3-D DFNs. The Glaerkin method has been successfully applied to solve the flow problem in single fracture planes [Koyama et al. 2009], rather than in fracture networks due to the difficulties of 
solving local mass conservation at the intersection of fractures and global mass conservation for flow continuity through the fracture networks. As mentioned in Section 3, each fracture plane is divided into several irregular regions. Each irregular region $R$ is bounded by circle edge $\Gamma_{f}$, intersection lines $S_{k}$ with other fractures and intersection lines with the domain faces. The local boundary condition on $S_{k}$ for each region $R$ can be written as $-K \partial h / \partial n_{k, f}=\beta_{k, f}$. The hydraulic head field in each element of region $R$ satisfies the formulation:

$$
\int_{\Omega^{(e)}}\left[\frac{\partial}{\partial x_{f}}\left(K \frac{\partial h}{\partial x_{f}}\right)+\frac{\partial}{\partial y_{f}}\left(K \frac{\partial h}{\partial y_{f}}\right)\right] \delta h d \Omega=0
$$

where $\delta h$ is a virtual quantity whose value will be zero on the constant hydraulic head boundary. Then using Gaussian formula the equation (6) can be written as

$$
\int_{\Omega^{(e)}} K \frac{\partial h}{\partial x_{f}} \cdot \frac{\partial \delta h}{\partial x_{f}}+K \frac{\partial h}{\partial y_{f}} \cdot \frac{\partial \delta h}{\partial y_{f}} d \Omega-\int_{S_{k}} \beta_{k, f} \cdot \delta h d S=0
$$

If an approximate solution using the Lagrange interpolation function is supposed on each triangle element

$$
\tilde{h}=\sum_{m=1}^{M} h_{m}^{(e)} \Phi_{m}^{(e)}
$$

where $\tilde{h}$ is the approximate solution of $h, m=1,2, \ldots M$ are nodes locally anticlockwise numbered for each element and for triangular mesh $M$ is equal to 3, superscript (e) represents the quantity on each element and $\Phi_{m}{ }^{(e)}$ is the interpolate function, or the shape function, with

$$
\Phi_{m}^{(e)}\left(x_{k n}\right)=\delta_{m n}= \begin{cases}1 & n=m \\ 0 & n \neq m\end{cases}
$$

and $\delta h=\Phi_{i}^{(e)}$, then substituting Eqs. (8) and (9) into Eq. (7) yields the flow equation on each element

$$
\sum_{n=1}^{M} h_{n}^{(e)}\left[\int_{\Omega^{(e)}}\left(K \cdot \frac{\partial \Phi_{m}^{(e)}}{\partial x} \cdot \frac{\partial \Phi_{n}^{(e)}}{\partial x}+K \cdot \frac{\partial \Phi_{m}^{(e)}}{\partial y} \cdot \frac{\partial \Phi_{n}^{(e)}}{\partial y}\right) \cdot d \Omega\right]=\int_{S_{k}} \beta_{k, f} \Phi_{m}^{(e)} d S
$$

Equation (10) can be rewritten in a matrix form as 


$$
\left[\begin{array}{ccc}
A_{11}^{(e)} & \cdots & A_{1 M}^{(e)} \\
\vdots & \vdots & \vdots \\
A_{M 1}^{(e)} & \cdots & A_{M M}^{(e)}
\end{array}\right]\left\{\begin{array}{c}
h_{1}^{(e)} \\
\vdots \\
h_{M}^{(e)}
\end{array}\right\}=\left\{\begin{array}{c}
F_{1}^{(e)} \\
\vdots \\
F_{M}^{(e)}
\end{array}\right\}
$$

where $A^{(e)}$ is the element stiffness matrix and $F^{(e)}$ represents the boundary conditions, with

$$
\begin{array}{r}
A_{m n}^{(e)}=\int_{\Omega^{(e)}}\left(K \cdot \frac{\partial \Phi_{m}^{(e)}}{\partial x} \cdot \frac{\partial \Phi_{n}^{(e)}}{\partial x}+K \cdot \frac{\partial \Phi_{m}^{(e)}}{\partial y} \cdot \frac{\partial \Phi_{n}^{(e)}}{\partial y}\right) \cdot d \Omega \\
F_{m}^{(e)}= \begin{cases}\int_{S_{k}} \beta_{k, f} \cdot \Phi_{m}^{(e)} d S & m \in S_{k} \\
0 & m \notin S_{k}\end{cases}
\end{array}
$$

The total stiffness matrix for each region is $\mathbf{A}_{i}=\sum A^{(e)}$. Then the coefficient matrix for fracture network can be written as

$$
\mathbf{A}=\left[\begin{array}{cc}
\tilde{\mathbf{A}} & \mathbf{A}_{12} \\
\mathbf{A}_{21} & \mathbf{0}
\end{array}\right]
$$

With

$$
\tilde{\mathbf{A}}=\left[\begin{array}{lll}
\mathbf{A}_{1} & & \mathbf{0} \\
& \ddots & \\
\mathbf{0} & & \mathbf{A}_{n}
\end{array}\right]
$$

The matrix $\mathbf{A}_{12}$ and $\mathbf{A}_{21}$ represent continuity conditions. Finally the problem can be reduced to solve the linear system

$$
[\mathbf{A}] \cdot\left[\begin{array}{c}
x_{N N} \\
X_{N I}
\end{array}\right]=\left[\begin{array}{c}
b_{N N} \\
\mathbf{0}
\end{array}\right]
$$

where $x_{N N}$ is the hydraulic heads on all nodes $N N, x_{N I}$ is the local flux on intersection nodes $N I$ and it is just an auxiliary variable, and $b_{N N}$ accounts for boundary conditions. The matrix $\mathbf{A}$ is symmetric and sparse. Either direct or iterative can be used to compute the solution. Then flow velocity on each element can be computed by differentiating the calculated hydraulic head. Total flux $Q$ through the model can be obtained by summing flow rate of each element on the outlet boundary. Finally, the equivalent permeability $K$ of the fracture network is computed according to: 


$$
Q=A \frac{K}{\mu} \frac{\Delta P}{L}
$$

where $A$ is the cross area, $L$ is the fracture network size and $\Delta P$ is the pressure difference between the inlet boundary and outlet boundary.

A MATLAB program is developed to model the fluid flow in 3-D DFNs. The flow computation code combined with other two modules (DFN modeling and Mesh generation) depicted in Fig. 2 constitute the complete flow computations in 3-D DFN models. The first module generates the fracture network using fractures following the well-known geometrical properties. A computational domain is defined, in which fracture segments outside the domain and the isolated ones that do no contribution to flow will be deleted. This module is accomplished based on the numerical software 3DEC. In the second module, the computational domain is firstly triangularly meshed in 3DEC. Then the grid points on fracture planes will be extracted and converted into Matlab input. Finally, the third module includes the global numbering of grid points, and identifying the intersection nodes and boundary nodes, and building the projection coordinate on each fracture. The flow computation would be accomplished by computing the local matrices in each fracture element and computing the global matrix and incorporating the boundary conditions and continuity conditions in global matrix and global right-hand side. The hydraulic head in each point and velocity in each triangle element would be obtained. Then the result would be checked by using a mass conservation verification. The computation is conducted only on the connected fracture planes without any dispose on fracture matrix which would largely reduce the computational costs. On a computational cluster with 64 CPUs, the efficacy of the code was demonstrated by a simulation time of about 4 min for case with fracture density of $0.1 \mathrm{~m}^{2} / \mathrm{m}^{3}$, a simulation time of about $2 \mathrm{~h}$ for case with fracture density of $0.4 \mathrm{~m}^{2} / \mathrm{m}^{3}$, and a simulation time of about $10 \mathrm{~h}$ for case with fracture density of $0.9 \mathrm{~m}^{2} / \mathrm{m}^{3}$. The total number of mesh grids for these three cases are 4693,23098 , and 61828, respectively. 


\section{Results and analysis}

\subsection{Verification of the proposed method}

A few 2-D and 3-D models are established to verify the validity the developed procedure. A simple parallel plate model (model I ) as shown in Fig. 3(a) with an aperture of $1 \mathrm{~mm}$ was firstly tested. According to the cubic law, the theoretical value of permeability, $k_{t}$, of the model can be obtained. The relative errors of numerical result of permeability, $k_{n}$, with respect to $k_{t}$ defined as $\varepsilon=$ $\left(k_{t}-k_{n}\right) / k_{t}$ are calculated. Results are listed in Table 1 . Then a configuration (model II) that contains two parallel plates dipping at $45^{\circ}$ and $135^{\circ}$ was tested. The aperture of each fracture was equal to 1 mm. Based on the study of Sarkar et al. [2004], the equivalent hydraulic aperture of an inclined parallel network can be determined by

$$
h_{e q}=\sqrt[3]{\sum_{i=1}^{n}\left(h_{i}^{3} \times \cos \theta_{i}\right)}
$$

where $h_{e q}$ is the equivalent aperture, $n$ is the total number of fractures, $h_{i}$ and $\theta_{i}$ are aperture and inclination angle of the $i^{\text {th }}$ fracture, respectively. As presented in Table 1 , the calculated results are in good agreement with the theoretical values for both single and intersecting parallel plates. Then a more complex configuration (model III) that contains five fractures as shown in Fig. 3(c) was tested. The equivalent hydraulic aperture of model III can also be calculated according to Eq. (16). The relative error of permeability between the simulation result and theoretical value is $0.067 \%$. Finally, the flow through a fracture with rough void field (model IV) is used to validate accurate approximation of influence of fracture heterogeneity on flow properties. Fig. 3(c) displays its tortuous fracture aperture field and the average magnitude is $3.87 \mathrm{~mm}$. Since it is difficult to obtain corresponding theoretical value, the numerical method to simulate the single rough fracture flow proposed by Koyama et al. [2009] is used as a contrast. The relative error of permeability is 3.63\%. This error is mainly attributed to the little difference of handling the element aperture between the two simulations. The proposed method is therefore basically reliable and is capable of analyzing the 
flow characteristics of 3-D DFN models.

\subsection{Permeability of 3-D DFN model and its 2-D cut planes}

The permeability of a 2-D cut plane is commonly considered as an approximation which can somewhat represents the "true" 3-D DFN permeability. This simplification would lead to an underestimation of fracture connectivity in 3-D DFN model. In order to quantify the underestimation, a series of 3-D DFN models is established with increasing fracture density from $0.1 \mathrm{~m}^{2} / \mathrm{m}^{3}$ to $0.9 \mathrm{~m}^{2} / \mathrm{m}^{3}$. A hydraulic aperture of $1 \mathrm{~mm}$ was assigned to all fractures. For each DFN model, a series of 11 rectangular cut planes are extracted. In this study only one flow direction parallel to the $x$-axis is considered, and the cut planes are all extracted parallel to the $x$ - $z$ plane as shown in Fig. 4.

Fig. 4 displays three examples of original 3-D DFN models for a low (Fig. 4(a)), medium (Fig. 4(b)), and high density (Fig. 4(c)) and cut planes (Fig. 4(d) (i)) extracted from them, correspondingly. In spite of the fact that all fractures in original models intersect directly or indirectly with inflow/outflow boundaries, there exist some isolated fractures or fracture clusters in their cut planes, and there may be even not a single continuous path formed between inlet and outlet boundary as shown in Figs. 4(d), (e) and (g). This phenomenon is relatively obvious with a low fracture density (Fig. 4(a)). Then the equivalent permeability of 2-D fracture network is evaluated using the method described in Liu et al. [2015].

The equivalent permeabilities computed from cut planes are lower than the permeability obtained from their original 3-D DFN model over the entire range of fracture density (Fig. 5). At a relative low density (i.e., $0.1 \mathrm{~m}^{2} / \mathrm{m}^{3}$ ), although there is flow through the 3-D model, none of their cut planes can form a continuous flow path between the inlet and outlet boundary. As the density increases, some cut-through flow paths start to be formatted however there still exist some impermeable cut planes (circled in Fig. 5). The permeabilities of those cut planes are dispersedly distributed and can vary within a range of five times. This variance would converge with the 
increasing fracture density. The average permeability of permeable cut planes would underestimate their 3-D counterpart by approximately from $19.2 \%$ to $43.6 \%$. This is limited for the 3-D DFN models with the density in a range of $0.1 \mathrm{~m}^{2} / \mathrm{m}^{3}$ to $0.9 \mathrm{~m}^{2} / \mathrm{m}^{3}$, below which the fractures may not be well connected to form flow paths and above which there are so many fractures that beyond the capability of a common computer. The underestimation comes from the structural limitation of 2-D analysis: the fractures which are not connected to the network in cut planes may be actually connected in the 3-D networks. The results are consistent with the study of Lang et al. [2014] who investigated the equivalent permeability tensor of fracture-matrix models. Therefore the fracture connectivity in 3-D DFN model cannot be accurately captured by their 2-D cut planes and it is necessary to conduct 3-D fracture network flow analysis.

\subsection{Effect of fracture length distribution}

The geometrical properties of fractures have significant influences on the fracture network topology and consequently influence its hydraulic behavior. Field observations have demonstrated that the fracture length follows power-law distributions, and the exponent varies extremely large from 2.0 to 4.5 [De Dreuzy, 2000]. To investigate the effect of fracture length distribution on fluid flow at a 3-D network scale, six DFN models that are consisted of fractures with power-law exponents of 2.0, 2.2, 3.0, 3.5, 4.0 and 4.5 are generated, respectively. The density $\mathrm{P}_{32}$ of each model is same and each fracture network contains four sets of fractures differing only in dip $\theta_{1}$ and dip direction $\theta_{2}$, as shown in Table 2. Fig. 6 displays three examples for a long ( $\left.a=2.0\right)$, medium ( $a$ $=3.0)$, and short $(a=4.5)$ fracture length. For a smaller $a$, the network structure is dominated by a few longer fractures. As a increases, the proportion of short fractures vs. long fractures increases, with the structure gradually dominated by the smaller fractures.

The equivalent permeability of the six DFN models is computed using the developed procedure. Results indicate that the permeability decreases exponentially with the increasing $a$ (see Fig. 7). The equivalent permeability for the models with $a=2.0$ is nearly five times larger than that 
with $a=4.5$. Since the fracture intersection plays a critical role on fracture connectivity, the average intersection length $L_{i}$, which defined as the ratio of total intersection length to total intersection number in each DFN model, is calculated and plotted in Fig. 8. Results show that $L_{i}$ decreases as $a$ increases, following a power-law equation. In DFN models with $a=4.5$, the number of short fractures is larger than that of long fractures. Hence, more fractures are generated to reach the same density for the models with a smaller $a$. This results in a larger number of intersection lines and a smaller $L_{i}$. In contrast, for DFN models with $a=2.0$, the network is dominated by long fractures. Its $L_{i}$ is much larger than that for DFN models with $\mathrm{a}=4.5$. Fracture intersection line is the only connection between two fractures and it has the determinant influence on fracture connectivity. Therefore, to some extent, the change of $L_{i}$ can account for the observed decrease of equivalent permeability in Fig. 7.

\subsection{Effect of aperture heterogeneity}

The 3-D DFN models mentioned above approximate all fracture planes with a same aperture value. In reality, the distribution of apertures in each single fracture is non-uniform, due to the roughness of the two facing rock walls. It was proved that the simplification for a flow in two smooth parallel plates overestimated the flow rate to a large extent [Brown 1987]. And it has been suggested that the fluid flow within a rough fracture should be characterized by formation of preferential flow paths [Brown 1987; Durham et al. 1997; Ishibashi et al. 2012]. In order to investigate the effect of aperture heterogeneity of each fracture plane, networks in which individual fractures have a realistic aperture distribution are established. The aperture fields are generated based on the shear of three artificial fracture specimens made in the study of Li et al. [2008]. These fracture specimens were manufactured from each of three natural fracture surfaces labeled as J1, J2

and J3, respectively. Among them, J1 is flat and has a few major asperities on its surface. The surface of $\mathrm{J} 2$ is smooth, however a major asperity exists at the center. J3 is very rough with no major asperities but a great number of small ones. The fractal dimension calculated for these 
fracture surfaces is 2.012, 2.025, and 2.066, respectively [Wang et al. 2014]. Fracture specimens are fully mated at initial condition with contact ratio very close to 1.0. Aperture field is abstracted at the shear displacement of $5 \mathrm{~mm}, 10 \mathrm{~mm}$ and $15 \mathrm{~mm}$ respectively for each of three specimens. This shearing is simulated by fixing the lower surface and moving the upper surface by a horizontal shear displacement, then uplifting the upper surface by $4 \mathrm{~mm}$ in order to eliminate the influence of overlapping rock mass into each other. Fig. 9 displays a statistics of aperture distribution at the shear displacement of 15 mm which can be closely modeled by a Gauss distribution.

The fracture apertures in 3-D networks (labeled as NJ1, NJ2, and NJ3) are assigned from aperture field of each of three fracture specimens at different shear displacements. Fig. 10 shows an example of aperture field extracted at the shear displacement of $15 \mathrm{~mm}$. Due to the difference of morphology of three fracture surfaces, the aperture fields change remarkably after a shear process. The surface of $\mathrm{J} 1$ is relatively smooth and flat, therefore the apertures of network NJ1 distribute evenly over the fracture plane. A major asperity in J2 leads to some obvious low-permeable areas for network NJ2. For network NJ3, the widely distributed asperities develop a very complex aperture field. A same topology fracture network with the homogeneous aperture of $4 \mathrm{~mm}$ is also generated as a comparison. Its aperture can be regarded as coming from a parallel specimen with fractal dimension equaling to 2.00 .

A hydraulic pressure difference of $0.01 \mathrm{MPa}$ is applied between the right and left boundary. The simulated flow velocities are superimposed in Fig. 10. Since there are three inlet ports on the right boundary, the flow rate of fractures near this side is smaller than that of left boundary. Results show that the flow patterns become more tortuous with the increasing fracture roughness. Especially in network NJ2 and NJ3, due to the complex structure of aperture fields, there form more obvious 3-D preferential flow paths comparing with the network NJ1. The fluid flow is mainly controlled by several preferential paths. This phenomenon of uneven flow is the channeling effect [Tsang and Tsang 1987]. At present only numerical simulation can illustrate realistically the flow localization (channeling) since it is difficult to conduct experiments with multiple-fracture sample. 
Fig. 11 shows the calculated equivalent permeability of DFNs in which fractures are characterized by aperture distribution abstracted from each of four fracture specimens under different shear displacements. For smooth fracture with fractal dimension $D$ equal to 2.00, there would lead to the same uniform aperture field at different shear displacement. Therefore, shear displacement has no effect on the calculated equivalent permeability. As $D$ increases from 2.00 to 2.02, the increasing shear displacement would lead to an increasing equivalent permeability. However, when $D$ is larger than 2.02, the magnitude of equivalent permeability tend to decrease with the increasing shear displacement. This phenomenon is mainly because of the stochastic distribution of the apertures in fractures. At the same displacement, a definite trend for equivalent permeability to decrease with an increasing fractal dimension is shown in Fig. 11 . The permeability for network NJ3 is approximately twice smaller than that for homogeneous one at shear displacement of $15 \mathrm{~mm}$, indicating that the permeability of natural roughness fracture networks cannot be properly predicted by a simulation where all fractures are modeled as parallel plates with the same aperture. This is mainly due to the fact that low transmissive areas induced by the aperture heterogeneity tend to break up flow paths then reduce its permeability. This is the purpose of the original that we tried to incorporate the aperture anisotropy into the 3-D DFNs to accurately estimate the permeability of networks with real geometrical properties.

\section{Conclusions}

This study presents a method to compute fluid flow through 3-D DFN models. The technique solves the Reynolds equations in each fracture by using the Galerkin method based on the continuity of mass and flux imposed at intersections between fractures. The method guarantees both local and global mass conservation, and can naturally incorporate geometrical properties in fracture and as a result can account explicitly for the contribution of aperture heterogeneity of individual fractures on fluid flow, which can be used to demonstrate the 3-D channeling flow. The validity of 
the method was validated for 3-D fracture models with constant and variable apertures, respectively. This approach is subsequently applied to compare the equivalent permeability of 3-D fracture networks with the permeabilities of the 2-D cut planes. The influences of fracture length and aperture heterogeneity on the network permeability were also estimated. Results show that, in spite of the fact that flow occurs through the 3-D DFN model, its 2-D cut planes may be totally impermeable with no continuous flow path formatted between the inlet and outlet boundaries. Whereas there may also exist some cut planes whose permeabilities are close to their 3-D counterpart. The permeabilities for those arbitrarily abstracted cut planes can vary within a range of five times and would converge with the increasing of fracture density. The average permeability of permeable cut planes would underestimate their 3-D counterpart by $19.2 \sim 43.6 \%$ for density varying from 0.1 to $0.9 \mathrm{~m}^{2} / \mathrm{m}^{3}$. The fracture connectivity/conductivity in 3-D DFN model hence cannot be accurately captured by their 2-D cut planes. With the increasing value of power-law exponent of length distribution function, the equivalent permeability would decrease exponentially within one order of magnitude. This phenomenon can be described by the average intersection length, a parameter that can reflect the fracture connectivity. The rougher fracture surfaces, the stronger anisotropy of aperture distribution, and thereby leading to the more tortuous flow channels in 3-D fracture networks. These results could provide a theoretical basis for quantitatively estimating behavior of shale gas migration and enhancing gas recovery in low permeability reservoirs.

In the future, the proposed procedure would be optimized to facilitate the solution of governing equations of fluid flow and improve the efficiency of calculation. The REV (representative elementary volume) of 3-D DFNs would be compared with that of 2-D models, and particle transport behavior in 3-D DFNs would be systematically investigated.

\section{Acknowledgments}

This study has been partially funded by National Natural Science Foundation of China (Grant 
No. 51379117) and China Scholarship Council (CSC). These supports are gratefully acknowledged.

\section{References:}

Andersson J, Dverstorp B. Conditional simulations of fluid flow in three - dimensional networks of discrete fractures. Water Resource Res, 1987, 23(10): 1876-1886.

Baecher G B, Lanney N A. Trace length biases in joint surveys//19th US Symposium on Rock Mechanics (USRMS). American Rock Mechanics Association, 1978.

Baghbanan A, Jing L. Hydraulic properties of fractured rock masses with correlated fracture length and aperture. Int $\mathrm{J}$ Rock Mech Min Sci, 2007, 44(5): 704-719.

Bear J, Tsang C F, De Marsily G. Flow and contaminant transport in fractured rock. Academic Press, 2012.

Benedetto M F, Berrone S, Borio A, Pieraccini S, Scialo S. A hybrid mortar virtual element method for discrete fracture network simulations. J Comput Phys, 2016, 306: 148-166.

Berkowitz B. Characterizing flow and transport in fractured geological media: A review. Adv Water Resour, 2002, 25(8): 861-884.

Bogdanov I I, Mourzenko V V, Thovert J F, Thovert J -F. Effective permeability of fractured porous media in steady state flow. Water Resource Res, 2003, 39(1).

Brown S R. Fluid flow through rock joints: the effect of surface roughness. J Geophys Res: Solid Earth, 1987, 92(B2): 1337-1347.

Cacas M C, Ledoux E, Marsily G, Barbreau A. Modeling fracture flow with a stochastic discrete fracture network: Calibration and validation: 2. The transport model. Water Resource Res, 1990, 26(3): 491-500.

Cai J, Sun S. Fractal analysis of fracture increasing spontaneous imbibition in porous media with gas-saturated. Int $\mathrm{J}$ Mod Phys C, 2013a, 24(08): 1350056.

Cai J, Guo S, You L, Hu X. Fractal analysis of spontaneous imbibition mechanism in fractured-porous dual media reservoir. Acta Phys. Sin., 2013b, 62(1): 014701. (in Chinese)

Cai J, Perfect E, Cheng C L, Hu X. Generalized modeling of spontaneous imbibition based on Hagen-Poiseuille flow in tortuous capillaries with variably shaped apertures. Langmuir, 2014, 30(18): 5142-5151.

Cai J, Luo L, Ye R, Zeng X, Hu X. Recent advances on fractal modeling of permeability for fibrous porous media. Fractals, 2015, 23(01), 1540006.

Chen Y F, Hu S H, Hu R, Zhou C B. Estimating hydraulic conductivity of fractured rocks from high-pressure packer tests with an Izbash’s law based empirical model. Water Resource Res, 2015b, 51(4): 2096-2118.

Darcel C, Bour O, Davy P. Stereological analysis of fractal fracture networks. J Geophys Res: Solid Earth, 2003, 108(B9).

Davy P, Darcel C, Bour O, Munier R, De Dreuzy J R. A note on the angular correction applied to fracture intensity profiles along drill core. J Geophys Res: Solid Earth, 2006, 111(B11).

De Dreuzy J R, Davy P, Bour O. Percolation parameter and percolation-threshold estimates for three-dimensional random ellipses with widely scattered distributions of eccentricity and size. Phys Rev E, 2000, 62(5): 5948.

De Dreuzy J R, Méheust Y, Pichot G. Influence of fracture scale heterogeneity on the flow properties of three - 
dimensional discrete fracture networks (DFN). J Geophys Res: Solid Earth, 2012, 117(B11).

De Dreuzy J R, Pichot G, Poirriez B, Erhel J. Synthetic benchmark for modeling flow in 3D fractured media. Comput Geosci, 2013, 50: 59-71.

Durham W B. Laboratory observations of the hydraulic behavior of a permeable fracture from $3800 \mathrm{~m}$ depth in the KTB pilot hole. J Geophys Res: Solid Earth, 1997, 102(B8): 18405-18416.

Erhel J, De Dreuzy J R, Poirriez B. Flow simulation in three-dimensional discrete fracture networks. SIAM J Sci Comput, 2009, 31(4): 2688-2705.

Fisher, N. I. Statistical Analysis of Circular Data. Cambridge University Press,1996.

Gonzalez - Garcia R, Huseby O, Thovert J F, et al. Three - dimensional characterization of a fractured granite and transport properties. J Geophys Res: Solid Earth, 2000, 105(B9): 21387-21401.

Hull L C, Miller J D, Clemo T M. Laboratory and simulation studies of solute transport in fracture networks. Water Resource Res, 1987, 23(8): 1505-1513.

Hyman J D, Karra S, Makedonska N, Gable C W, Painter S L, Viswanathan H S. dfnWorks: A discrete fracture network framework for modeling subsurface flow and transport. Comput Geosci, 2015, 84: 10-19.

Ishibashi T, Watanabe N, Hirano N, Okamoto A, Tsuchiya N. GeoFlow: A novel model simulator for prediction of the 3 - D channeling flow in a rock fracture network. Water Resource Res, 2012, 48(7).

Ivanova V M, Sousa R, Murrihy B, Einstein H H. Mathematical algorithm development and parametric studies with the GEOFRAC three-dimensional stochastic model of natural rock fracture systems. Comput Geosci, 2014, 67: 100-109.

Jing Z, Willis - Richards J, Watanabe K, Hashida T. A three - dimensional stochastic rock mechanics model of engineered geothermal systems in fractured crystalline rock. J Geophys Res: Solid Earth, 2000, 105(B10): 23663-23679.

Khamforoush M, Shams K, Thovert J F, Adler P M. Permeability and percolation of anisotropic three-dimensional fracture networks. Phys Rev E, 2008, 77(5): 056307.

Koudina N, Garcia R G, Thovert J F, Adler P M. Permeability of three-dimensional fracture networks. Phys Rev E, 1998, 57(4): 4466.

Koyama T, Li B, Jiang Y, Jing L. Numerical modelling of fluid flow tests in a rock fracture with a special algorithm for contact areas. Comput Geotech, 2009, 36(1): 291-303.

Lang P S, Paluszny A, Zimmerman R W. Permeability tensor of three - dimensional fractured porous rock and a comparison to trace map predictions. J Geophys Res: Solid Earth, 2014, 119(8): 6288-6307.

Lee C I, Song J J. Rock engineering in underground energy storage in Korea. Tunn Undergr Sp Tech, 2003, 18(5): 467-483.

Lei Q, Latham J P, Tsang C F, et al. A new approach to upscaling fracture network models while preserving geostatistical and geomechanical characteristics. J Geophys Res: Solid Earth, 2015, 120(7): 4784-4807.

Li B, Jiang Y, Koyama T, Jing L, Tanabashi Y. Experimental study of the hydro-mechanical behavior of rock joints using a parallel-plate model containing contact areas and artificial fractures. Int J Rock Mech Min Sci, 2008, 45(3): 362-375.

Liu R, Jiang Y, Li B, Wang X. A fractal model for characterizing fluid flow in fractured rock masses based on randomly distributed rock fracture networks. Comput Geotech, 2015, 65: 45-55.

Liu R, Li B, Jiang Y. A fractal model based on a new governing equation of fluid flow in fractures for characterizing 
hydraulic properties of rock fracture networks. Comput Geotech, 2016a, 75: 57-68.

Liu R, Li B, Jiang Y. Critical hydraulic gradient for nonlinear flow through rock fracture networks: The roles of aperture, surface roughness, and number of intersections. Adv Water Resour, 2016b, 88: 53-65.

Long J, Gilmour P, Witherspoon P A. A Model for Steady Fluid Flow in Random Three - Dimensional Networks of Disc - Shaped Fractures. Water Resource Res, 1985, 21(8): 1105-1115.

Min K B, Jing L, Stephansson O. Determining the equivalent permeability tensor for fractured rock masses using a stochastic REV approach: method and application to the field data from Sellafield, UK. Hydrogeol J, 2004, 12(5): 497-510.

Mustapha H, Mustapha K. A new approach to simulating flow in discrete fracture networks with an optimized mesh. SIAM J Sci Comput, 2007, 29(4): 1439-1459.

Nick H M, Paluszny A, Blunt M J, Matthai S K. Role of geomechanically grown fractures on dispersive transport in heterogeneous geological formations. Phys Rev E, 2011, 84(5): 056301.

Neuman S P. Trends, prospects and challenges in quantifying flow and transport through fractured rocks. Hydrogeol J, 2005, 13(1): 124-147.

Noetinger B. A quasi steady state method for solving transient Darcy flow in complex 3D fractured networks accounting for matrix to fracture flow. Journal of Computational Physics, 2015, 283: 205-223.

Rutqvist J, Leung C, Hoch A, Wang Y, Wang Z. Linked multicontinuum and crack tensor approach for modeling of coupled geomechanics, fluid flow and transport in fractured rock. Journal of Rock Mechanics and Geotechnical Engineering, 2013, 5(1): 18-31.

Sarkar S, Toksoz M N, Burns D R. Fluid flow modeling in fractures. Massachusetts Institute of Technology. Earth Resources Laboratory, 2004.

Tsang Y W, Tsang C F. Channel model of flow through fractured media. Water Resource Res, 1987, 23(3): 467-479.

Wang G, Huang N, Jiang Y, Li B, Wu Xuezhen, Zhang X. Seepage calculation model for rough joint surface considering fractal characteristics. Chinese Journal of Rock Mechanics and Engineering , 2014, 33(Supp.2): 3397-3405. (in Chinese)

Wei W, Cai J, Hu X, Fan P, Han Q, Lu J, Cheng CL, Zhou F. A numerical study on fractal dimensions of current streamlines in two-dimensional and three-dimensional pore fractal models of porous media. Fractals, 2015, 23(01): 1540012.

Zhang Q H, Yin J M. Solution of two key issues in arbitrary three-dimensional discrete fracture network flow models. J Hydrol, 2014, 514: 281-296.

Zhang Q H. Finite element generation of arbitrary 3-D fracture networks for flow analysis in complicated discrete fracture networks. J Hydrol, 2015, 529: 890-908.

Zhao Z, Jing L, Neretnieks I, Moreno L. Numerical modeling of stress effects on solute transport in fractured rocks. Comput Geotech, 2011, 38(2): 113-126. 


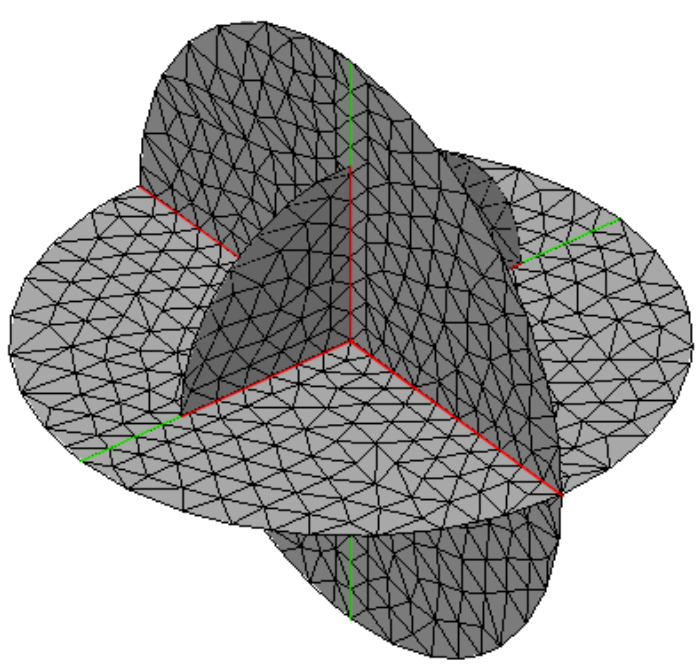

Fig. 1 An example of three intersecting fractures with a triangular mesh: red lines are actual intersection lines, and the green lines are their corresponding extensions. 


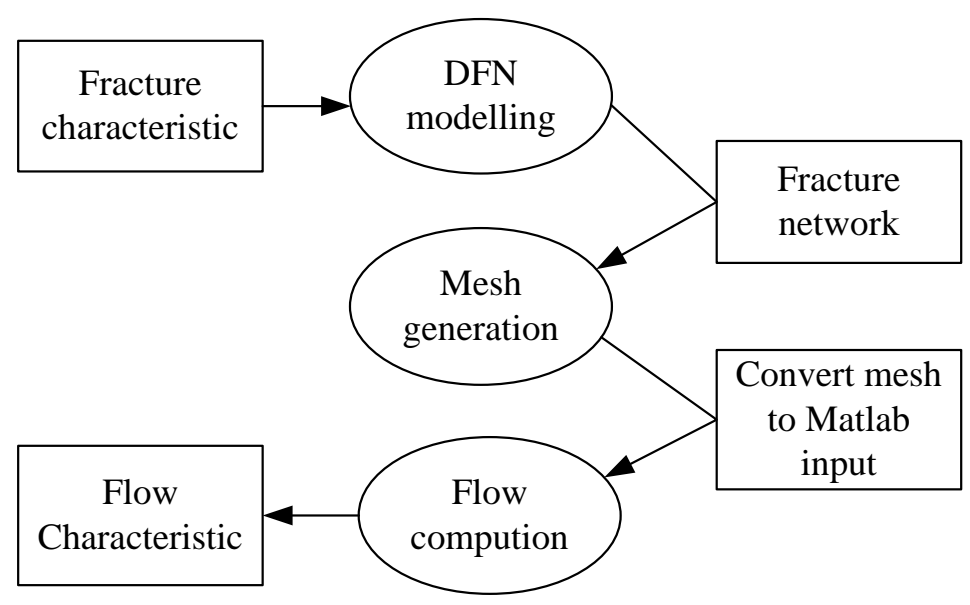

Fig. 2 Computational modules and main process for flow in 3-D DFN model. 
(a)

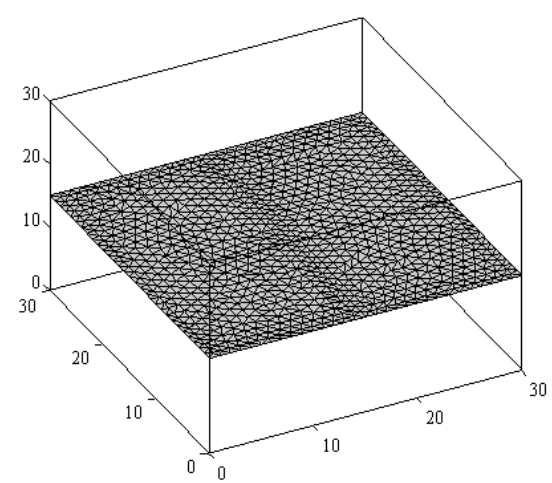

(b)

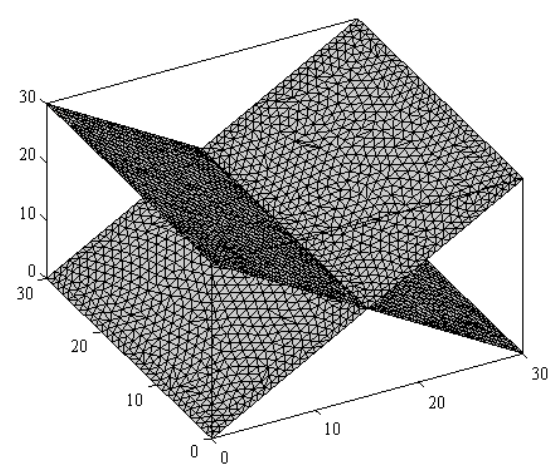

(c)

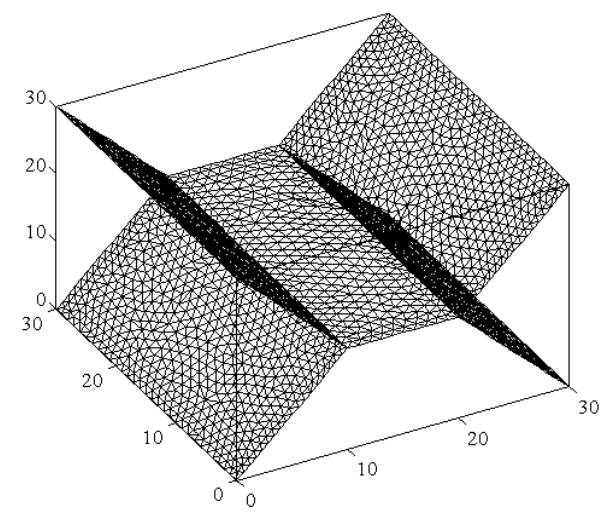

(d)

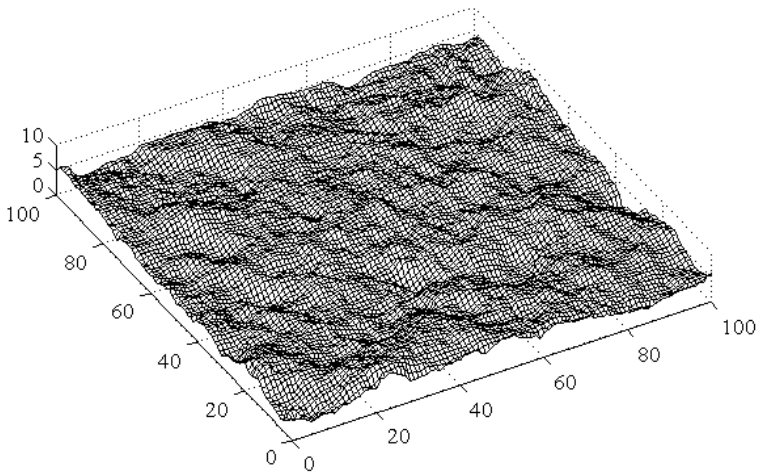

Fig. 3 Verification models for developed procedure: (a) model I: single parallel plate fracture; (b) model II: two intersecting fractures; (c) model III: five intersecting fractures; (d) model IV: single fracture with heterogeneous apertures. 

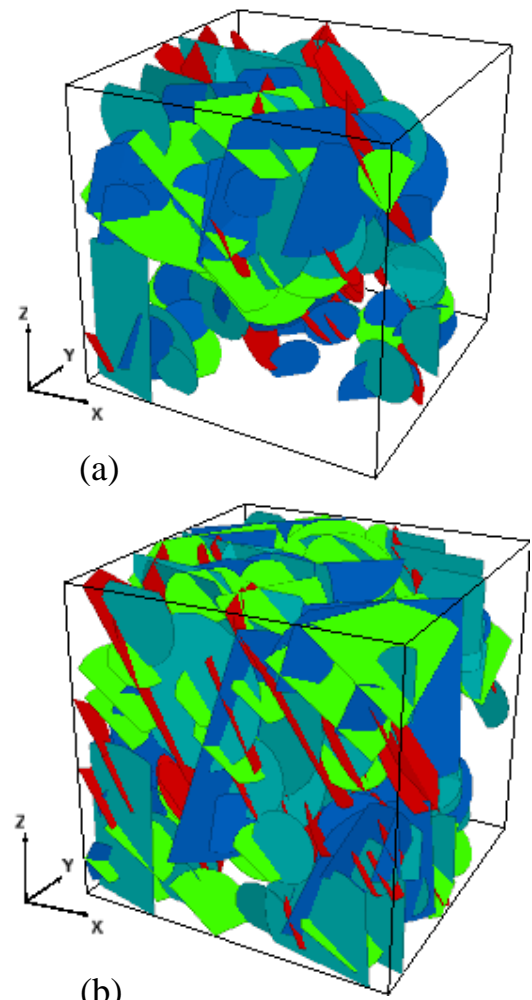

(b)

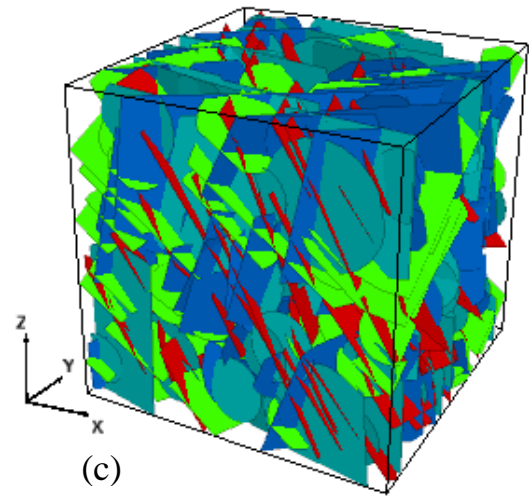

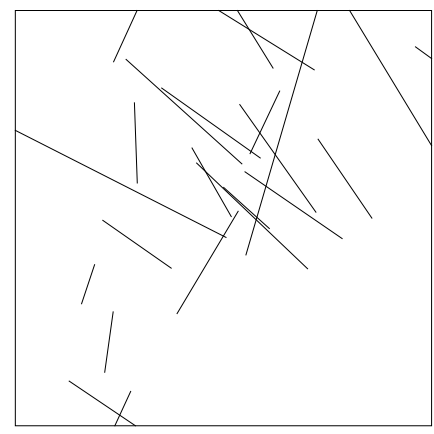

(d)

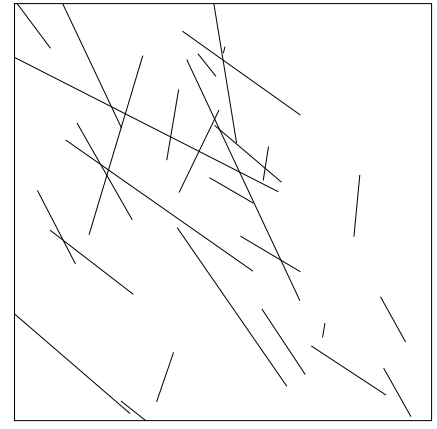

(g)

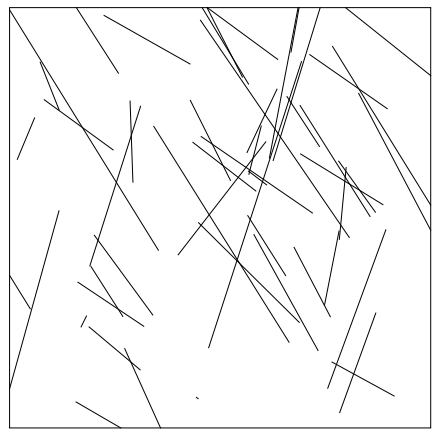

(e)

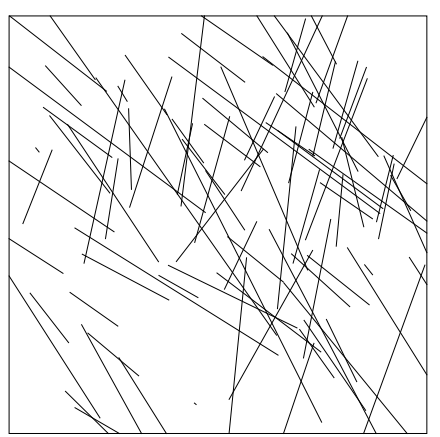

(f)

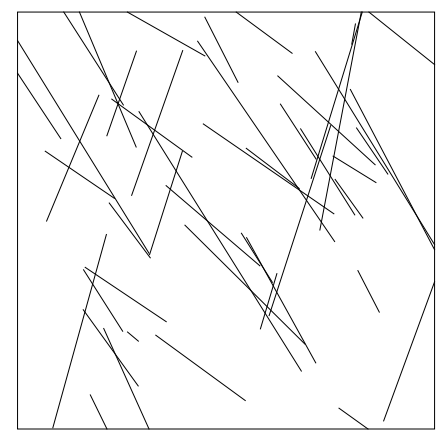

(h)

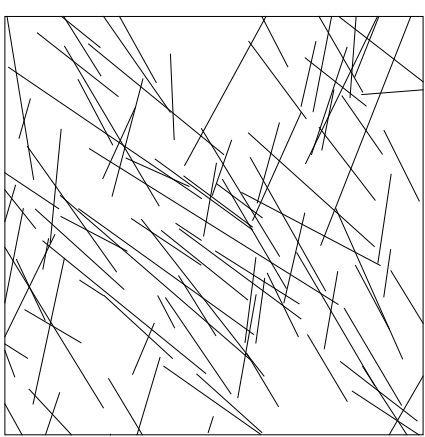

(i)

Fig. 4 Examples of original 3-D DFN models for a (a) Low, (b) medium, (c) high fracture density and cut planes (d) (i) extracted from each. 


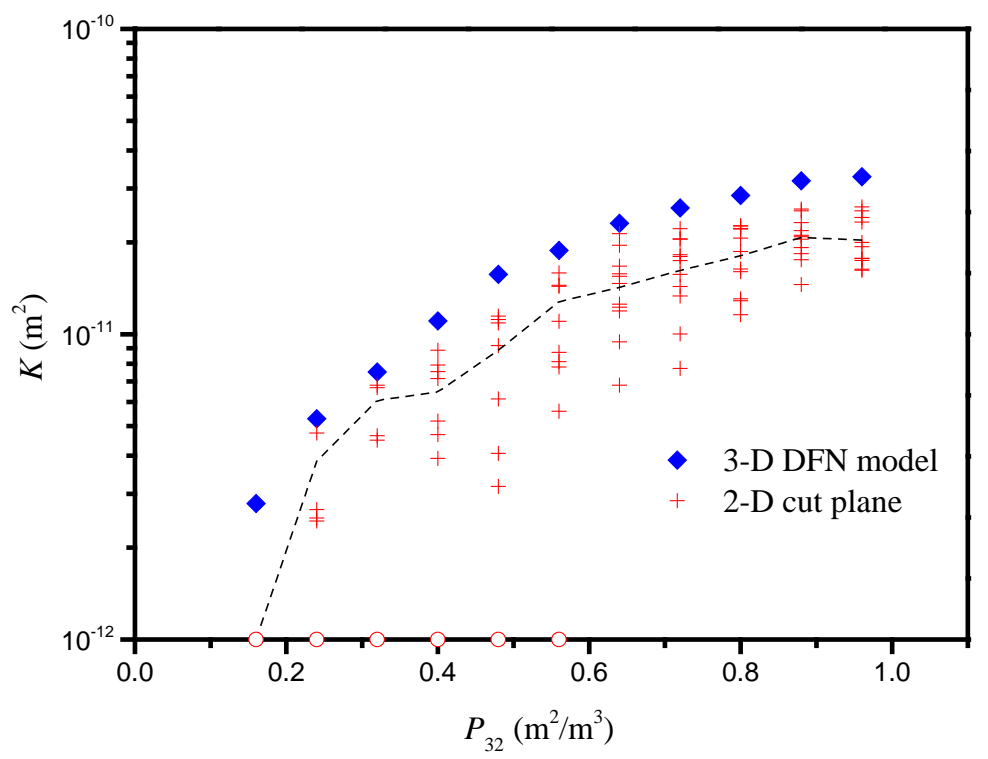

Fig. 5 Equivalent permeability of 3-D DFN models and their 2-D cut planes. Note that the circled points indicate some impermeable cut planes and dotted line indicates the average value for those permeable cut planes. 

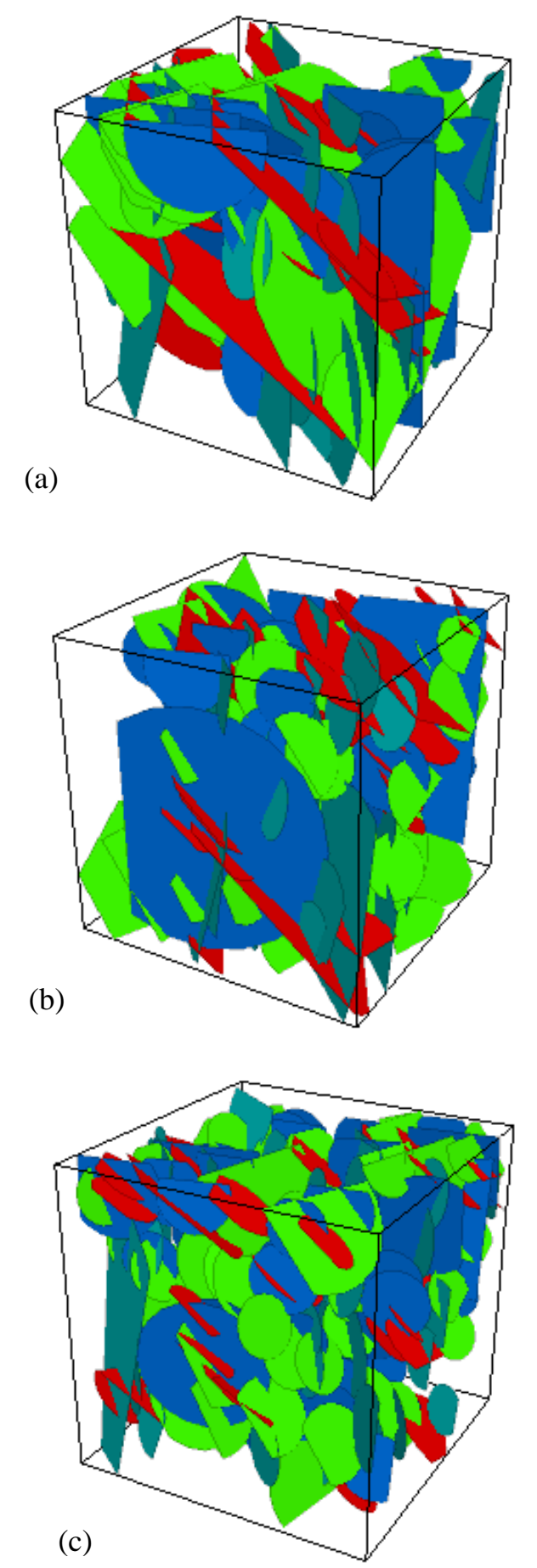

Fig. 6 Typical fracture networks for power-law length exponents of (a) $a=2.0$, (b) $a=3.0$ and (c) $a=4.5$. 


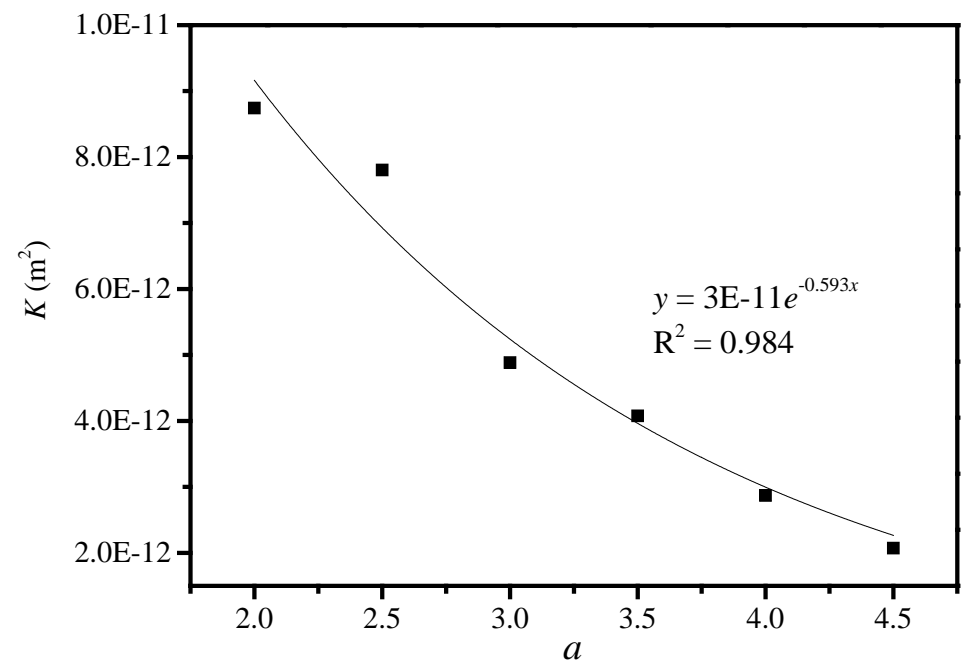

Fig. 7 Relationship between equivalent permeability $K$ and power-law exponent $a$. 


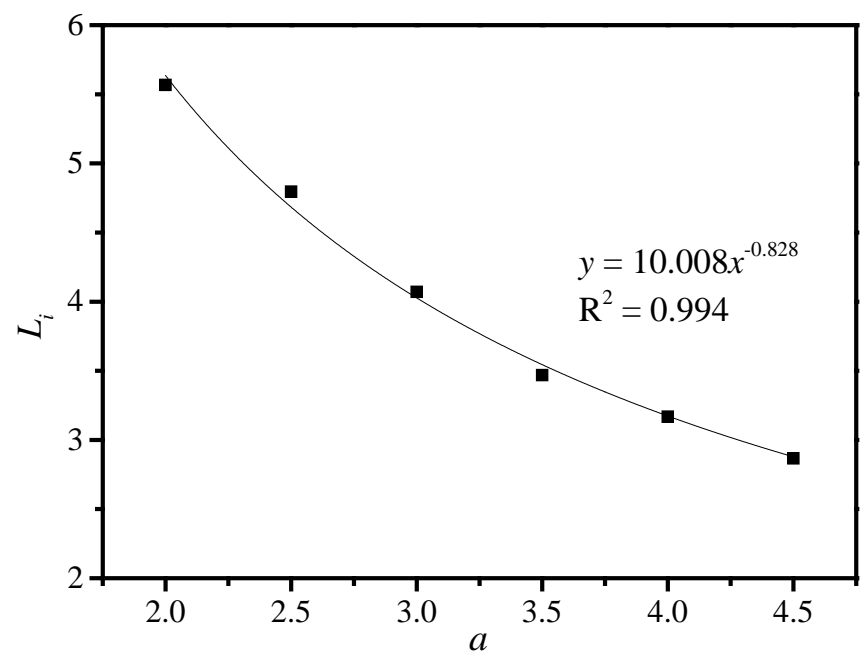

Fig. 8 Relationship between the average intersection length $L_{i}$ and power-law exponent $a$. 

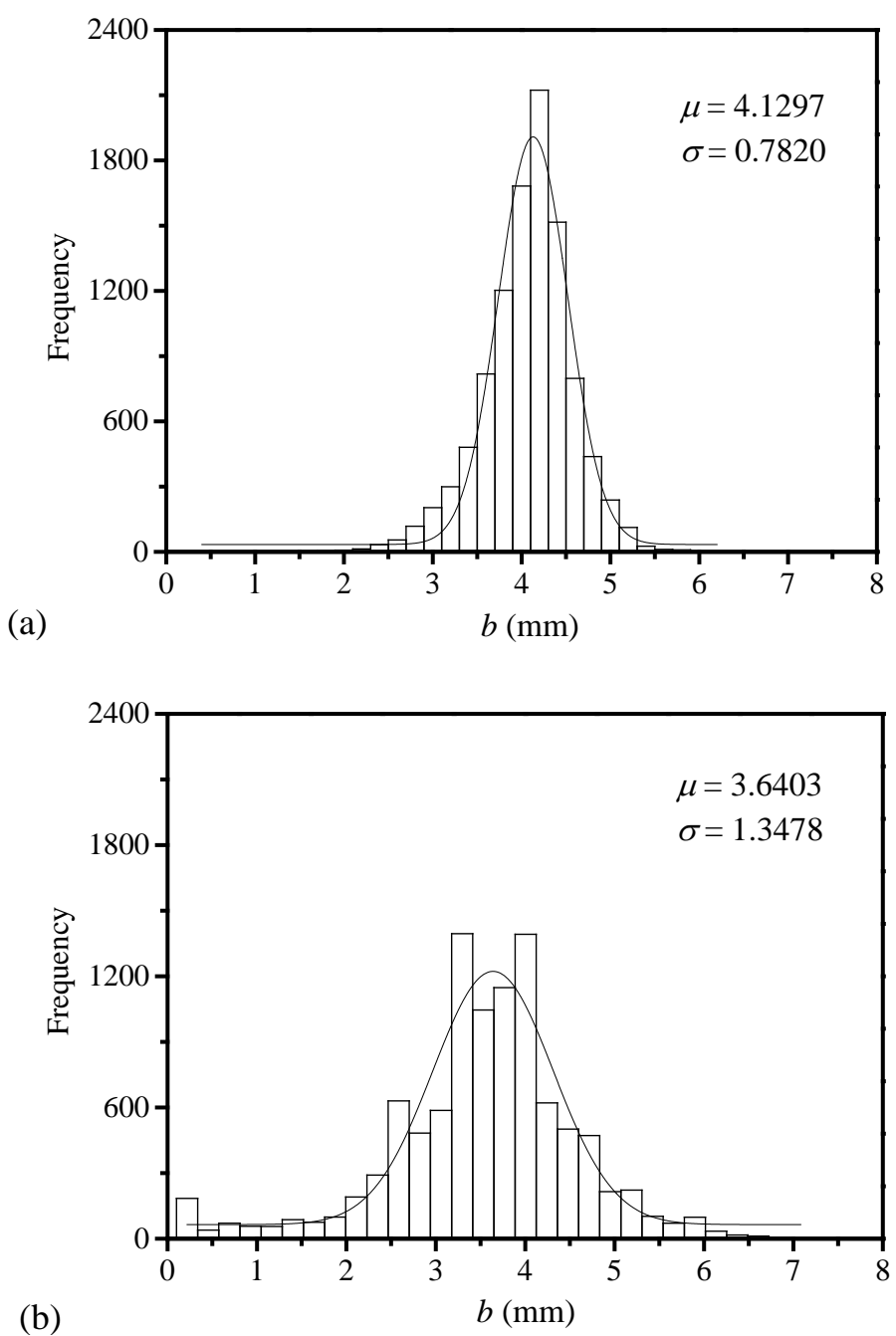

(b)

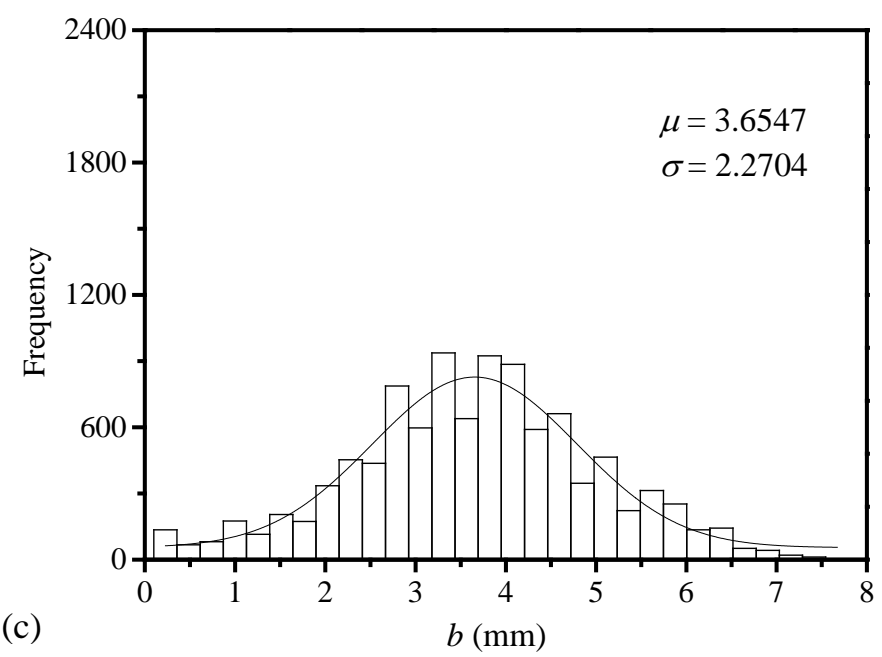

Fig. 9 Histograms and distributions for the aperture $b$ of: (a) J1, (b) J2 and (c) J3. 
(a)

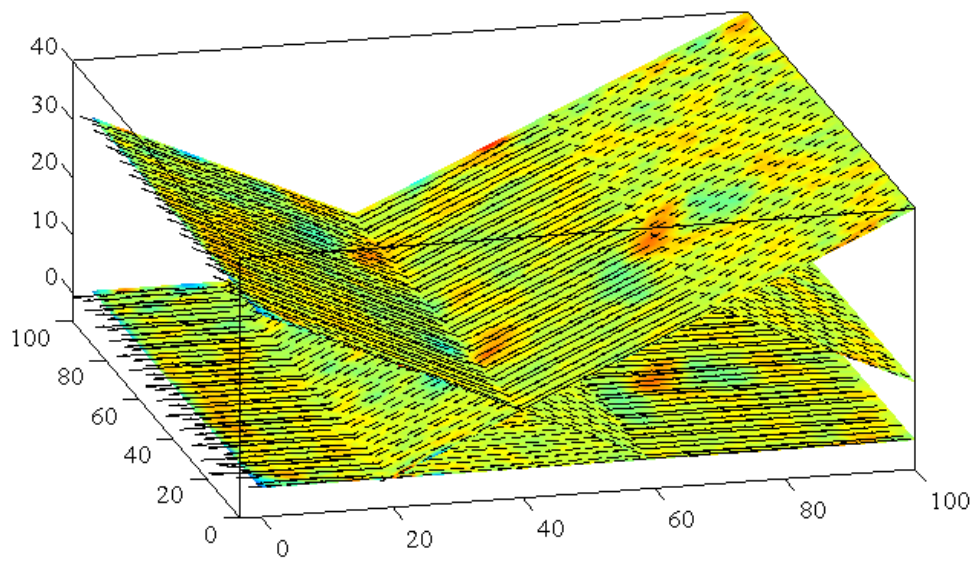

(b)

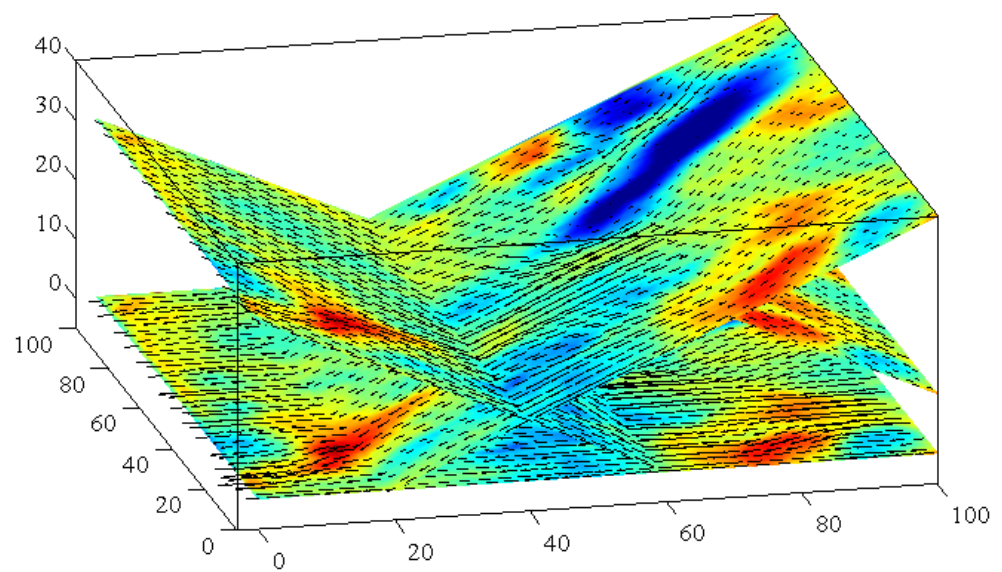

(c)
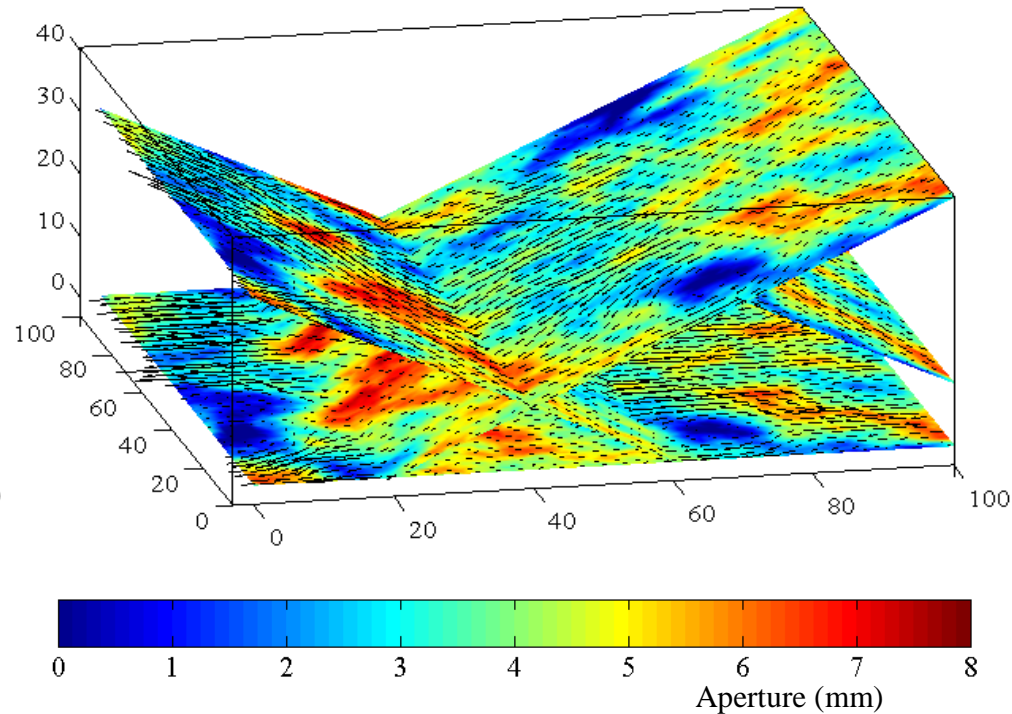

Fig. 10 Local fracture aperture distribution superimposed with flow velocity fields for networks: (a) NJ1, (b) NJ2 and (c) NJ3. 


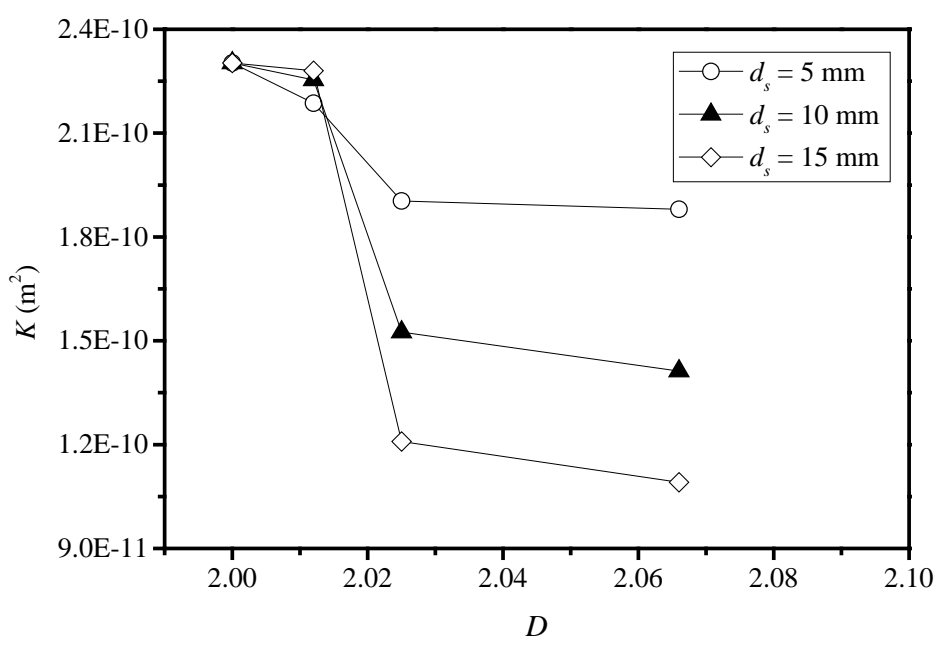

Fig. 11 Equivalent permeability of DFNs in which fractures are characterized by aperture distribution abstracted from each of four fracture specimens under different shear displacement. 
Table 1 Comparisons between theoretical values and numerical results.

\begin{tabular}{cccc}
\hline Model & $\mathrm{k}_{\mathrm{t}}\left(m^{2}\right)$ & $\mathrm{k}_{\mathrm{s}}\left(m^{2}\right)$ & $\varepsilon$ \\
\hline I & $2.778 \mathrm{E}-12$ & $2.778 \mathrm{E}-12$ & 0 \\
II & $3.928 \mathrm{E}-12$ & $3.930 \mathrm{E}-12$ & $0.051 \%$ \\
III & $2.973 \mathrm{E}-12$ & $2.975 \mathrm{E}-12$ & $0.067 \%$ \\
IV & $4.377 \mathrm{E}-10$ & $4.218 \mathrm{E}-10$ & $3.633 \%$ \\
\hline
\end{tabular}


Table 2 Parameters used for the generation of DFNs.

\begin{tabular}{lll}
\hline Property & Distribution & Parameters \\
\hline $\begin{array}{l}\text { Domain size }(\mathrm{m}) \\
\text { Positions }\end{array}$ & Uniform & \\
Orientations, $\left(^{\circ}\right)$ & Fisher & Set1: $\theta_{1}=90, \theta_{2}=10, \kappa=200$ \\
& & Set2: $\theta_{1}=40, \theta_{2}=120, \kappa=200$ \\
& & Set3: $\theta_{1}=60, \theta_{2}=70, \kappa=200$ \\
& & Set $4: \theta_{1}=80, \theta_{2}=320, \kappa=200$ \\
& & $2<a<4.5,0.167<l / L<1$ \\
Length & Power law & $P_{32}=0.4$ \\
\hline
\end{tabular}

